\title{
FREE BOUNDARY PROBLEMS FOR THE UNSTEADY TRANSONIC SMALL DISTURBANCE EQUATION: TRANSONIC REGULAR REFLECTION
}

\author{
SUNČICA ČANIĆ* $*$ BARBARA LEE KEYFITZ ${ }^{\dagger}$, AND EUN HEUI KIM ${ }^{\ddagger}$
}

\begin{abstract}
We formulate and solve a transonic regular reflection problem for the unsteady transonic small disturbance equation, using a free boundary problem approach. Our method applies to self-similar shock reflection when the incident shock angle is large enough to permit a regular reflection configuration with a subsonic state behind the reflected shock. For the small-disturbance approximation in weak shock reflection, this corresponds to relatively large wedge angles. One contribution of this paper is the development of an asymptotic formula for the reflected shock, far from the reflection point, and for the subsonic state far downstream. These asymptotic series are valid for the small-disturbance approximation, for any incident shock angles.

The main result in the paper is an existence theorem for the nonuniform subsonic flow behind the reflected shock. The flow velocity satisfies a quasilinear elliptic equation which is coupled to the Rankine-Hugoniot equations for the reflected shock, forming a free boundary problem on part of the boundary. Because the equation is not uniformly elliptic in the entire domain, we introduce a cut off to give a bounded domain, and also cut offs to the coefficients.

Our result is incomplete in the following sense: we have been unable to remove the cut offs entirely. However, we prove that the flow we have constructed solves the original problem in a domain of finite size around the reflection point.
\end{abstract}

1. Introduction. This paper is inspired by work of Cathleen Morawetz, [17], which analyses the bifurcation patterns in shock reflection by a wedge. Morawetz considers weak shocks and small wedge angles, for which the transonic full potential equation provides a good model, as entropy and vorticity vanish to third order in the shock strength. In the shock interaction region, the equation reduces further to the unsteady transonic small disturbance (UTSD) equation, the model we consider here. Our results are complementary to those in [17]: While that paper establishes values of the wedge angle parameter at which regular or Mach reflection occurs, we consider only the range in which regular reflection is expected to occur. While Morawetz identifies two types of regular reflection, weak and strong, and shows that entropy considerations favor the occurrence of weak reflection, we focus on transonic reflection, which is the strong case except for a narrow range of wedge angles near the transition, where both the weak and the strong reflection are transonic. Finally, while Morawetz assumes the existence of nonconstant subsonic flows, we verify this assumption in the particular case we study.

For technical reasons involving the theory of oblique derivative boundary value problems in elliptic equations, we are restricted to using the UTSD equation, although there is no reason to suppose these results could not be extended to other elliptic problems such as the subsonic full potential equation. However, to our knowledge the machinery is not yet in place for this.

Analysis near the shock interaction point is one component of Morawetz's contribution in [17]. A second important feature, which is adapted in our paper, is the

\footnotetext{
* Department of Mathematics, University of Houston, Houston, Texas 77204-3476, USA. Research supported by the National Science Foundation, grant DMS-9970310.

${ }^{\dagger}$ Department of Mathematics, University of Houston, Houston, Texas 77204-3476, and Brown University, Providence, Rhode Island, 02912, USA (keyfitz@uh.edu). Research supported by the Department of Energy, grant DE-FG-03-94-ER25222, and by the National Science Foundation, grant DMS-9973475 (POWRE).

$\ddagger$ Department of Mathematics, University of Houston, Houston, Texas 77204-3476, USA.
} 

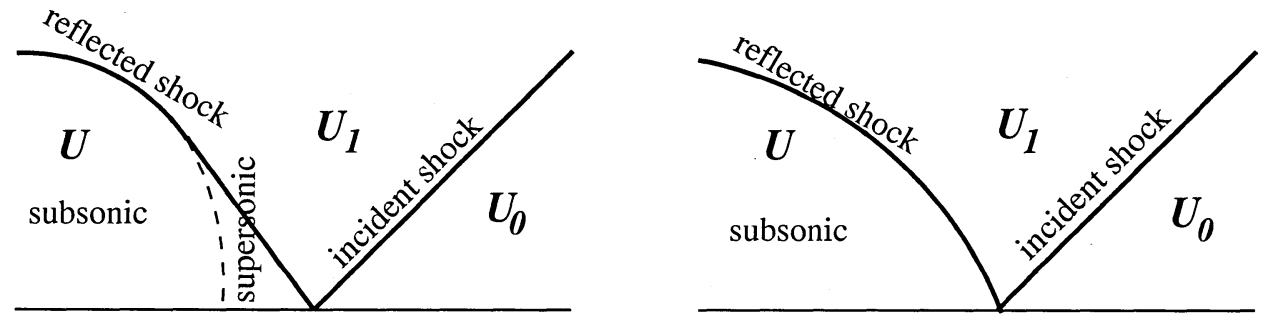

FIG. 1.1. Supersonic and Transonic Regular Reflection

asymptotic analysis of the reflected shock, far from the interaction point. Morawetz showed that the reflected shock is "almost semi-circular", and almost sonic. In our case, since we use the UTSD equation throughout the flow field, we obtain an "almost parabolic" and almost sonic shock asymptotically. Unlike the problem in [17], the UTSD model gives rise to an unbounded subsonic region. The asymptotic information is useful in choosing boundary conditions when we cut off the region to obtain a bounded domain.

1.1. Self-Similar Problems in Shock Reflection. When one attempts to solve two-dimensional self-similar problems in similarity variables, there results a system of conservation laws which changes type. In the simplest case, of a system of two equations, the system is hyperbolic outside the region of complicated interactions, and elliptic in the "subsonic" region in which waves from different sources interact.

A number of difficulties must be overcome to produce a complete solution to even the simplest Riemann problem, for even the simplest equation. The difficulties include solving the Cauchy problem in the hyperbolic region for a system of conservation laws with a source term and with coefficients which involve the independent variables. A second problem is determining the position of the sonic line, at which the equations change type. Then, one may have to solve a degenerate quasilinear elliptic equation of Keldysh type. Finally, the boundary value problem in the subsonic region, for a typical problem which contains shocks, will present the transonic shock position as a free boundary.

In earlier papers, $[4,5,6,7,9]$, we have looked at these problems separately. In this paper, we prove the following theorem.

THEOREM 1.1. A solution $U=(u, v)$ to the UTSD equation, (2.1), in the upper half-plane, satisfying the conditions for regular reflection and with the property that the flow behind the reflected shock is fully subsonic, exists in a finite neighborhood of the reflection point, $\left(\xi_{a}, 0\right)$. The solution is a function of the similarity variables, $(\xi, \eta)=(x / t, y / t)$; it satisfies the boundary conditions $v(\xi, 0)=0$ on the symmetry boundary $\eta=0$ (at the wall), $U=U_{0}=(0,0)$ for $\xi>a \eta+\xi_{a}$ (ahead of the incident shock) and $U=U_{1}=(1,-a)$ in the region between the incident and the reflected shocks. For all $a \geq \sqrt{2}$ such a solution exists and contains a curved reflected shock. If $\sqrt{2} \leq a \leq a^{*}$, there are two such solutions (the "strong" and the "weak" solution).

See Figure 1.1 for a sketch of the geometry. The UTSD equation is given, and other terms defined, in Section 2.

This paper, while it still does not produce a complete solution, advances the program in two ways: 

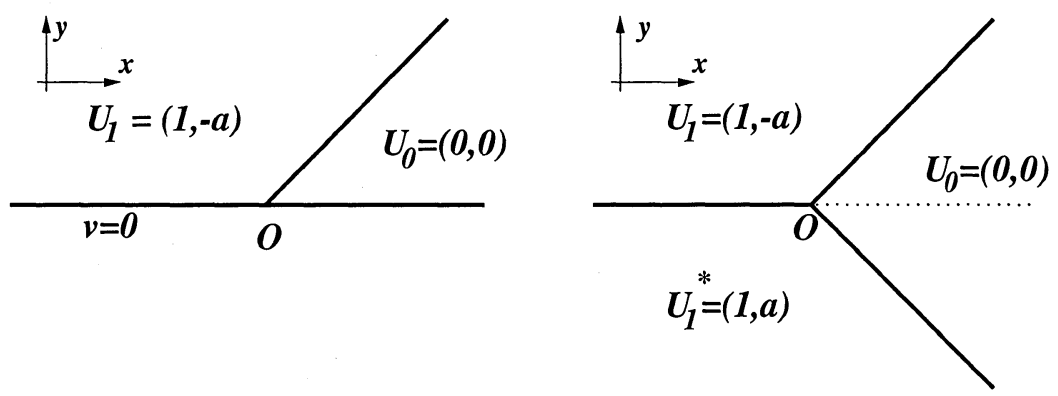

FIG. 1.2. Riemann Data for the Shock Reflection Problem

1. We obtain an equation for the asymptotic position of a reflected shock for a Riemann problem with shock reflection data (as distinct from interaction of two independent shocks). In this way we are able to extend the class of problems to which our earlier results [7, 9] apply by explicitly including shock reflections.

2. We show that the free boundary problem for one kind of shock reflection, the completely transonic case, has a solution. Our method is based on the technique of Canić, Keyfitz and Lieberman, [8], which we extend from the steady to the unsteady small disturbance equation, and from a perturbation result, in which the solution is confined to a small neighborhood of a constant state.

However, our result is incomplete in the following sense: In order to use a fixed point method to solve the free boundary problem, we have had to assume certain a priori bounds on the solution. We cannot, at present, verify all these bounds except in a finite neighborhood of the shock interaction point. Thus we have constructed only a local solution, near the shock interaction point. We have not proved that a global solution exists (although numerical evidence points this way), or that our solution coincides with the global solution near the interaction point. Despite this difficulty, we are presenting our partial results because the technique appears promising. We also hope that other researchers may have insights into how to extend this result.

1.2. Different Types of Regular Reflection. Our work on Riemann problems for the UTSD equation, [7], predicts that there will be two types of regular shock reflection patterns, depending on whether the flow behind the reflected wave is subsonic everywhere or not.

We remark that the work in [7] concerns shock interaction problems, in which the Riemann data consist of three piecewise constant states in the upper half-plane, separated by discontinuities which evolve as shocks. The corresponding full-plane problem has four states, separated by four shocks, and a symmetry condition. In this paper, we look at shock reflection data, which lead to a simpler problem and are closer to the motivating problem of shock reflection by a wedge. Figure 1.2 sketches two ways of portraying shock reflection Riemann data: as a half-plane problem with a symmetry boundary condition at the wall or as a full-plane, three-state Riemann problem.

If there is a supersonic (hyperbolic) region behind the reflected wave, near the point where the incident and the reflected waves meet at the wall, then the reflected shock is a straight line near the reflection point. On the other hand the flow im- 
mediately behind the reflected wave may be subsonic (elliptic). In either case, the reflected wave has a well-defined angle of reflection; see Figure 1.1. Both cases occur (see [7]): if $a>\sqrt{2}$ then there are two possible reflected states at the reflection point, $U_{R}=\left(u_{R}, 0\right)$ and $U_{F}=\left(u_{F}, 0\right)$. The larger value, $U_{F}$, is always subsonic, and $U_{R}$ is also subsonic if $a<a^{*} \approx 1.45$. (These states are computed in Section 2.1.)

Reflected waves of both types are seen in the numerical simulations of Canić and Mirković, [10]. For a given incident shock angle, the two types of regular reflection are known as "weak" and "strong"; the term "strong" refers to the larger value, $U_{F}$. This nomenclature is used by Morawetz in [17], which uses the shock polars for the UTSD equation, and goes back to von Neumann. Ben-Dor, [1, page 21], calculates weak and strong regular reflections for the compressible Euler equations, and notes that the tangency at the shock polar (where the two states coalesce) is not at the sonic point, so the same phenomenon, of a small range of incident shock angles in which both weak and strong reflections are subsonic, occurs also in the full model.

When there is no supersonic region behind the reflected wave, then the equations behind the reflected shock are strictly elliptic, and one technique for proving existence of a solution is to adapt the free boundary approach of Canić, Keyfitz and Lieberman, [8]. We take a first step toward carrying this out in the present paper. To use the free boundary technique of [8], we introduce several cut off functions. We are not able to remove these modifications completely, and thus we have not proved that our solution to the modified problem solves the original problem, except near the reflection point. With this proviso, in the case $\sqrt{2}<a<a^{*}$, we obtain two solutions this way. If $a>a^{*}$, then we obtain only the fully transonic solution; we conjecture that a second solution, which is supersonic behind the shock and becomes transonic further down, will be found by adapting the technique here to handle the degenerate elliptic equation which governs the subsonic flow in that case. However, we leave this for another paper. A second question, not resolved in this paper, is that of uniqueness of the solution. The purely self-similar method of this paper does not distinguish between admissible and inadmissible solutions. The analogy with one-dimensional problems is that if one solves Riemann problems by using the Rankine-Hugoniot relation, without regard to satisfying the Lax geometric entropy condition, one will get more than one solution to a number of problems.

In the next section, we give the equations and develop the asymptotics for the problem; in Section 3 we state the main theorem precisely and in Section 4 we give the proof. We discuss removing the cut off functions in Section 5 .

2. Background. In this section, we assemble the equations and review the reductions used in the remainder of the paper. We derive an asymptotic result on the transonic shock position in Section 2.3, and on the boundary condition on the cut off domain boundary in Section 2.4.

It is worth stressing that two kinds of cut offs are used in this paper: We truncate the subsonic domain to a bounded domain, and we introduce cut offs in the coefficients of the equations to keep them strictly elliptic or to enforce other bounds. The first kind of cut off, as mentioned in our earlier papers on the UTSD equation [7, 9], is necessary in order to deal with a peculiarity of this equation, that the subsonic region is always unbounded. This difficulty disappears in, for instance, the nonlinear wave equation or the full potential equation. However, the nonlinear wave equation is not a model for weak shock reflection, and the full potential equation, used by Morawetz in [17] away from the shock, does not permit a formulation in which we can use the elliptic theory required here. The work of Brio and Hunter [2,3] and Tabak and 
Rosales [18] develops theoretical background and computational results on the UTSD equation model for weak shock reflection. The asymptotic results in Sections 2.3 and 2.4 are used to resolve the difficulty of the unbounded domain. Then in the remainder of the paper we restrict attention to a bounded domain, which we call $\Omega$.

The cut offs which we introduce to ensure uniform ellipticity, boundedness of the state $u$, obliqueness of the free boundary condition, and existence of the mapping defining the free boundary, are another matter. Only if we eliminate these functions can we claim to have found a global solution. As stated before, we have not completed this. We show, in Section 5, that we can eliminate one cut off function and that our solution is strictly elliptic in the subsonic region. However, a second cut off is only partially removed. At the moment, we do not know if this difficulty can be overcome, or if there are in some cases obstructions to finding a solution this way.

2.1. Some Useful Formulas. The governing partial differential equation, the unsteady transonic small disturbance (UTSD) equation, in self-similar coordinates $\xi=x / t, \eta=y / t$, is

$$
\begin{aligned}
(u-\xi) u_{\xi}-\eta u_{\eta}+v_{\eta} & =0 \\
-v_{\xi}+u_{\eta} & =0
\end{aligned}
$$

It is convenient to work in another coordinate system, $(\rho, \eta)$, with $\rho=\xi+\eta^{2} / 4$. In the $(\rho, \eta)$ coordinate system, the self-similar UTSD equation becomes

$$
\begin{aligned}
(u-\rho) u_{\rho}-\frac{\eta}{2} u_{\eta}+v_{\eta} & =0 \\
\frac{\eta}{2} u_{\rho}-v_{\rho}+u_{\eta} & =0
\end{aligned}
$$

Eliminating $v$ yields the second-order equation

$$
Q(u)=\left((u-\rho) u_{\rho}+\frac{u}{2}\right)_{\rho}+u_{\eta \eta}=0
$$

with quasilinear form

$$
Q(u)=(u-\rho) u_{\rho \rho}+u_{\eta \eta}+u_{\rho}^{2}-\frac{1}{2} u_{\rho}=0 .
$$

The ellipticity ratio is

$$
\frac{\Lambda}{\lambda}=\frac{\max \{u-\rho, 1\}}{\min \{u-\rho, 1\}} .
$$

When the domain $\Omega$ is bounded, the ratio is estimated by

$$
\frac{\Lambda}{\lambda} \leq \frac{\max |\rho|+\max |u|}{\min (u-\rho)}
$$

assuming $u-\rho>0$. For the shock reflection problem, the incident shock,

$$
S_{1}: \xi=a \eta+\omega_{1} \quad \text { with } \quad \omega_{1}=\frac{1}{2}+a^{2},
$$

separates the ambient (upstream) state $U_{0}=(0,0)$ from the state $U_{1}=(1,-a)$ behind the incident shock. See Figure 1.2. This normalization is now standard for the UTSD 
equation; see Brio and Hunter, [2], for example. When $a \geq \sqrt{2}$, the reflected states (solutions to the quasi-one-dimensional Riemann problem $[6,7]$ ) are

$$
U_{R}=\left(1+a^{2}-a \sqrt{a^{2}-2}, 0\right) \quad \text { or } \quad U_{F}=\left(1+a^{2}+a \sqrt{a^{2}-2}, 0\right) .
$$

The point on the wall where incident and reflected shocks meet, in a regular reflection configuration, is

$$
\Xi_{a}=\left(\omega_{1}, 0\right)=\left(\frac{1}{2}+a^{2}, 0\right)=\left(\xi_{a}, 0\right) .
$$

If the reflected shock were rectilinear then its equation would be $\xi=\kappa \eta+\omega$, where $\omega_{R}=\omega_{F}=\omega_{1}=\frac{1}{2}+a^{2}$ and $\kappa=\kappa_{R}$ or $\kappa=\kappa_{F}$ :

$$
\kappa_{R}=-\frac{1}{a-\sqrt{a^{2}-2}} \quad \text { and } \quad \kappa_{F}=-\frac{1}{a+\sqrt{a^{2}-2}} .
$$

A state $U=(u, v)$ is super- or subsonic at $(\xi, \eta)$ according as $\xi+\eta^{2} / 4-u>0$ or $<0$. The state $U_{F}$ is always subsonic at $\Xi_{a}$, while $U_{R}$ is also subsonic if $a<a^{*}$. A simple calculation, [7], shows that $a^{*}=(1+\sqrt{5} / 2)^{1 / 2}>\sqrt{2}$. When $a^{2}$ is close to 2 , both $\kappa_{R}$ and $\kappa_{F}$ are close to $-1 / a \approx-1 / \sqrt{2} \approx-.7$. There are two solutions to this problem; as stated in the Introduction, an admissibility condition arising from a more complete (for example, fully time dependent) theory would be needed to resolve the nonuniqueness.

By causality, if the flow is subsonic immediately behind the shock, then it remains so, and the reflected shock remains transonic downstream. If the horizontal flow velocity is also bounded, by $u_{\max }$ say, then the shock cannot cross the sonic parabola corresponding to $u_{\max }, \xi+\eta^{2} / 4=u_{\max }$, and hence is curved. An asymptotic description of the shock, given in Section 2.3, shows it tends to $P_{1}=\left\{\xi+\eta^{2} / 4-1=0\right\}$ (the sonic line for $U_{1}$ ) as $\xi \rightarrow-\infty$. Our construction in the subsonic region is consistent with this assumption.

2.2. Rankine-Hugoniot Conditions along the Reflected Shock. The Rankine-Hugoniot equations are

$$
\frac{d \xi}{d \eta}=-\frac{[v]}{[u]}=\frac{\left[\frac{1}{2} u^{2}-\xi u\right]}{[v-\eta u]}
$$

where the square brackets, [ ], denote jumps across the shock $\xi(\eta)$. Solving for the wave of interest (with negative slope), we have

$$
\begin{aligned}
& \xi^{\prime}=-\frac{\eta}{2}-\sqrt{\xi+\eta^{2} / 4-(u+1) / 2} \\
& \xi^{\prime}=-(v+a) /(u-1) .
\end{aligned}
$$

As in [8], we use both Rankine-Hugoniot conditions to obtain a free boundary problem with oblique derivative boundary data along the free boundary, and an evolution equation for the shock. The evolution of $\xi$ is given by (2.5). Eliminating $\xi^{\prime}$ in (2.5) and (2.6), we obtain an algebraic equation in $u$ and $v$ :

$$
v+a=(u-1)(\eta / 2+\sqrt{\rho-(u+1) / 2}),
$$

where we are using the notation $\rho=\xi+\eta^{2} / 4$. 
2.2.1. Conditions Expressed in $\rho, \eta$-Coordinates. In this paper, we solve the subsonic and free-boundary problems in terms of $(\rho, \eta)$ rather than $(\xi, \eta)$. We rewrite the equations, for reference. The Rankine-Hugoniot equations are now expressed as

$$
\frac{d \rho}{d \eta}=\frac{\left[\frac{1}{2} u^{2}-\rho u\right]}{\left[v-\frac{\eta}{2} u\right]}=\frac{\left[\frac{\eta}{2} u-v\right]}{[u]}
$$

from which we obtain the first Rankine-Hugoniot condition, equivalent to (2.5),

$$
\rho^{\prime}=-\sqrt{\rho-\frac{u+1}{2}} .
$$

A second equation is obtained by differentiating (2.7) along $\rho(\eta)$, and using the partial differential equation (2.2), so the left side of (2.7) has the derivative

$$
\begin{aligned}
v^{\prime}=\rho^{\prime} v_{\rho}+v_{\eta}=\rho^{\prime}\left(\frac{\eta}{2} u_{\rho}+u_{\eta}\right)+\left(\frac{\eta}{2} u_{\eta}-(u-\rho) u_{\rho}\right) & \\
& =\left(\rho^{\prime} \frac{\eta}{2}-(u-\rho)\right) u_{\rho}+\left(\rho^{\prime}+\frac{\eta}{2}\right) u_{\eta}
\end{aligned}
$$

and the derivative of the right side of (2.7) (here and in the remainder of the paper $\bar{u}=(u+1) / 2)$, since $u^{\prime}=\rho^{\prime} u_{\rho}+u_{\eta}$, is

$$
\begin{aligned}
& u^{\prime}\left(\frac{\eta}{2}+\right.\sqrt{\rho-\bar{u}})+(u-1)\left(\frac{1}{2}+\frac{\rho^{\prime}-\frac{1}{2} u^{\prime}}{2 \sqrt{\rho-\bar{u}}}\right) \\
&=\left(\frac{\eta}{2}+\sqrt{\rho-\bar{u}}-\frac{u-1}{4 \sqrt{\rho-\bar{u}}}\right) \rho^{\prime} u_{\rho}+\left(\frac{\eta}{2}+\sqrt{\rho-\bar{u}}-\frac{u-1}{4 \sqrt{\rho-\bar{u}}}\right) u_{\eta} \\
& \quad+\frac{(u-1)}{2}\left(1+\frac{\rho^{\prime}}{\sqrt{\rho-\bar{u}}}\right) .
\end{aligned}
$$

Equating the two expressions derived from (2.7) gives

$$
\begin{aligned}
\left(\rho^{\prime} \frac{\rho-\frac{1}{4}(3 u+1)}{\sqrt{\rho-\bar{u}}}+u-\rho\right) u_{\rho}+\left(\frac{\rho-\frac{1}{4}(3 u+1)}{\sqrt{\rho-\bar{u}}}-\rho^{\prime}\right) & u_{\eta} \\
& =-\frac{(u-1)}{2}\left(1+\frac{\rho^{\prime}}{\sqrt{\rho-\bar{u}}}\right) .
\end{aligned}
$$

This is equivalent to (2.7). If we now use (2.8) to substitute $-\rho^{\prime}$ for $\sqrt{\rho-\bar{u}}$, multiply the equation by $\rho^{\prime} / 2$, and finally substitute $\rho-\bar{u}$ for $\left(\rho^{\prime}\right)^{2}$, we obtain

$$
\beta \cdot \nabla u=0, \text { where } \beta=\left(\beta^{1}, \beta^{2}\right)=\left(\rho^{\prime}\left[\frac{7 u+1}{8}-\rho\right], \frac{5 u+3}{8}-\rho\right) .
$$

The operations which derived equations (2.8) and (2.9) from (2.4) can be reversed, so (2.8) and (2.9) are equivalent to the original Rankine-Hugoniot equations. We state this for reference.

Proposition 2.1. If $(u, v)$ is a solution to the self-similar UTSD equation (2.2) up to a discontinuity $\rho(\eta)$ which satisfies (2.8) and (2.9), and if the Rankine-Hugoniot equations (2.4) are satisfied at one point, then the equations (2.4) are satisfied everywhere along the discontinuity. 
Equation (2.9) is one of several alternate formulations of the oblique derivative boundary condition. Since $\nu=\left(-1, \rho^{\prime}\right) / \sqrt{1+\left(\rho^{\prime}\right)^{2}}$ is the inner unit normal to the subsonic domain along the shock $\rho(\eta)$, we compute

$$
\beta \cdot \nu=\frac{\beta \cdot\left(-1, \rho^{\prime}\right)}{\sqrt{1+\left(\rho^{\prime}\right)^{2}}}=-\frac{\rho^{\prime}(u-1)}{4 \sqrt{1+\left(\rho^{\prime}\right)^{2}}},
$$

and observe that this is nonnegative if $u \geq 1$. This expression must be compared to $|\beta|$ to evaluate the obliqueness constant. Adopting the definition in (2.9) for $\beta$, we see that $|\beta|$ is bounded if $\rho$ and $u$ are bounded. Later in the paper, we will show that we can bound $u-1$ away from zero, and, by introducing a cut off function, we will bound $\left|\rho^{\prime}\right|$ away from zero to obtain uniform obliqueness.

2.3. The Asymptotic Position of the Reflected Shock. We wish to formulate a free boundary problem for the reflected shock, and also a mixed boundary value problem for regions with fixed boundaries consisting of approximate shock positions. As a preliminary calculation, we show that the position of the reflected shock can be given asymptotically at a large distance from the origin. The development here does not constitute a proof that any reflected shock must have this position. Rather, it gives us a place to look for it; if we prove existence of a solution in a cut off region of the shape predicted by the asymptotics, then this reinforces the asymptotic prediction.

Far downstream, we postulate a single transonic shock, $\xi(\eta)$, connecting $U_{1}=$ $(1,-a)$ with a subsonic state $U=(u, v)$. Now, $\xi$ will lie between $P_{1}=\left\{\xi+\eta^{2} / 4-1=\right.$ $0\}$ and another parabola $P=\left\{\xi+\eta^{2} / 4-c=0\right\}$ for some constant $c>1$, so we can write the shock as

$$
\xi(\eta)=-\frac{\eta^{2}}{4}+1+b(\eta)
$$

where $b>0$ is bounded; we form the ansatz $b \rightarrow 0$ as $\eta \rightarrow \infty$.

To establish that this behavior is consistent with the Rankine-Hugoniot conditions, we expand $b, u$ and $v$ along the shock in powers of $1 / \eta$. Write

$$
u(\eta)=1+\theta(\eta) .
$$

From (2.10), $d \xi / d \eta=-\eta / 2+b^{\prime}$, and from the substitutions (2.10) and (2.11), we have

$$
\xi+\frac{\eta^{2}}{4}-\frac{u+1}{2}=b-\frac{\theta}{2}
$$

Hence the first Rankine-Hugoniot equation, (2.5), is equivalent to

$$
b^{\prime}=-\sqrt{b-\frac{\theta}{2}} \quad \text { or } \quad\left(b^{\prime}\right)^{2}=b-\frac{\theta}{2} .
$$

We use the other Rankine-Hugoniot relation in the form

$$
[v]=-\xi^{\prime}[u] \quad \text { or } \quad[v]=\eta \frac{\theta}{2}-\theta b^{\prime}
$$

from (2.4). Now we expand all the variables in asymptotic series about $\eta=\infty$ :

$$
[v]=\sum_{0}^{\infty} \frac{v_{i}}{\eta^{i}}, \quad \theta=\sum_{1}^{\infty} \frac{\theta_{i}}{\eta^{i}}, \quad b=\sum_{1}^{\infty} \frac{b_{i}}{\eta^{i}}, \quad b^{\prime}=-\sum_{1}^{\infty} \frac{i b_{i}}{\eta^{i+1}} .
$$


LEMMA 2.2. We can find the sequences of coefficients $\left\{\theta_{i}\right\}$ and $\left\{b_{i}\right\}$ recursively in terms of the sequence $\left\{v_{i}\right\}$.

For shock admissibility, we require $[u]=\theta>0$ and $\xi^{\prime}<0$, so $[v]>0$ and hence $v_{0}>0$. Other than that, the coefficients $v_{i}$ may take any values. As $\eta \rightarrow \infty$, $v \rightarrow-a+v_{0}$ may be positive, negative or zero. The value $v_{0}=a>0$ gives $v=0$ below the shock, a particularly attractive condition if one conjectures that the subsonic solution approaches a constant at downstream infinity. Note that although $[u] \rightarrow 0$ as $\eta \rightarrow \infty,[v]$ does not approach zero.

Proof. Substitute the power series in (2.14) into the second expressions in (2.12) and (2.13). Write (2.13) as $\eta \theta / 2=[v]+\theta b^{\prime}$, or

$$
\sum_{1}^{\infty} \frac{\theta_{i}}{2 \eta^{i-1}}=\sum_{0}^{\infty} \frac{v_{i}}{\eta^{i}}-\left(\sum_{1}^{\infty} \frac{\theta_{\ell}}{\eta^{\ell}}\right)\left(\sum_{1}^{\infty} \frac{j b_{j}}{\eta^{j+1}}\right) .
$$

Expanding gives

$$
\begin{aligned}
\frac{1}{2} \theta_{1}+\frac{1}{2} \frac{\theta_{2}}{\eta}+\frac{1}{2} \frac{\theta_{3}}{\eta^{2}} & +\ldots+\frac{1}{2} \frac{\theta_{j}}{\eta^{j-1}}+\ldots=v_{0}+\frac{v_{1}}{\eta}+\frac{v_{2}}{\eta^{2}}+\ldots+\frac{v_{j}}{\eta^{j}}+\ldots \\
& -\frac{\theta_{1} b_{1}}{\eta^{3}}-\frac{\theta_{2} b_{1}+2 \theta_{1} b_{2}}{\eta^{4}}-\frac{f_{3}\left(\left\{\theta_{3}\right\},\left\{b_{3}\right\}\right)}{\eta^{5}}-\ldots-\frac{f_{j}\left(\left\{\theta_{j}\right\},\left\{b_{j}\right\}\right)}{\eta^{j+2}}
\end{aligned}
$$

where $f_{j}\left(\left\{\theta_{j}\right\}\right)$ is an expression involving terms of the series truncated at the $j$-th term. Thus, $\theta_{1}=2 v_{0}, \theta_{2} / 2=v_{1}, \theta_{3} / 2=v_{2}, \theta_{4} / 2=v_{3}-\theta_{1} b_{1}$, and so on. Similarly, expand (2.12):

$$
\sum_{1}^{\infty} \frac{b_{i}}{\eta^{i}}-\sum_{1}^{\infty} \frac{\theta_{i}}{2 \eta^{i}}=\left(-\sum_{1}^{\infty} \frac{j b_{j}}{\eta^{j+1}}\right)\left(-\sum_{1}^{\infty} \frac{\ell b_{\ell}}{\eta^{\ell+1}}\right)
$$

or

$$
\begin{aligned}
\frac{b_{1}-\frac{\theta_{1}}{2}}{\eta}+\frac{b_{2}-\frac{\theta_{2}}{2}}{\eta^{2}}+\frac{b_{3}-\frac{\theta_{3}}{2}}{\eta^{3}}+\frac{b_{4}-\frac{\theta_{4}}{2}}{\eta^{4}}+\ldots \\
=\frac{b_{1}^{2}}{\eta^{4}}+\frac{4 b_{1} b_{2}}{\eta^{5}}+\frac{6 b_{1} b_{3}+4 b_{2}^{2}}{\eta^{6}}+\frac{g_{4}\left(\left\{b_{4}\right\}\right)}{\eta^{7}}+\ldots
\end{aligned}
$$

Thus, $b_{1}-\theta_{1} / 2=0, b_{2}-\theta_{2} / 2=0, b_{3}-\theta_{3} / 2=0, b_{4}-\theta_{4} / 2=b_{1}^{2}$, and so on. For any choices of $\left\{v_{i}\right\}$, we select

$$
\begin{array}{llll}
\theta_{1}=2 v_{0}, & \theta_{2}=2 v_{1}, & \theta_{3}=2 v_{2}, & \theta_{4}=2 v_{3}-2 \theta_{1} b_{1}=2 v_{3}-4 v_{0}^{2}, \\
b_{1}=\frac{\theta_{1}}{2}=v_{0}, & b_{2}=\frac{\theta_{3}}{2}=v_{1}, & b_{3}=\frac{\theta_{3}}{2}=v_{2}, & b_{4}=\frac{\theta_{4}}{2}+b_{1}^{2}=v_{3}-v_{0}^{2},
\end{array}
$$

and so on. Since the nonlinear expressions always involve previously determined factors, we can solve recursively for all $\theta_{j}$ and $b_{j}$.

2.4. Phragmen-Lindelöf Type Estimates for the Solution. If a function satisfies a linear elliptic partial differential equation in an unbounded domain and is known to be bounded, then one can get precise estimates on the bound and on behavior at infinity, based on the boundary conditions on the finite parts of the boundary. This is a type of generalization of the maximum principle. Gårding, [11], derives the result and gives the estimates for harmonic functions. These estimates are often called Phragmen-Lindelöf type estimates. 
Here we obtain similar estimates for the quasilinear equation (2.3). The basic construction uses separated solutions for the linear equation; we conjecture that such solutions will give bounds in our case also. This could be proved by using sub- and supersolutions; however, since we are using this development only to motivate our choice of a lower bound for $u-1$, we omit the proofs.

We consider equation (2.3), $Q(u)=0$, in $(\rho, \eta)$ coordinates. The domain is, roughly, the half plane $\rho<1$ (see Figure 3.1). Translating $\rho$, we look for bounds in a domain of the form $\left\{\rho^{2}+\eta^{2}>1\right\} \cap\{\rho<0\}$. Assuming $u$ to be bounded, we can linearize $Q(u)$. Neglecting all lower order terms, we obtain the equation

$$
-\rho u_{\rho \rho}+u_{\eta \eta}=0
$$

The change of variable $x=2 \sqrt{-\rho}$ now gives us $\Delta u=0$ (again, ignoring lower order terms), in the domain $\left\{x^{2}+\eta^{2}>1\right\} \cap\{x>0\}$. Since, our solution $u$ is close to the value unity on the straight boundary, we translate $u$ to $u-1$. Then the boundary conditions are $u(0, \eta)=0$, and $u=C$ on the curved boundary. A separated solution in polar coordinates is $u=C \cos \theta / r$, which gives the estimate

$$
u=\frac{C}{r} \cos \theta=\frac{C x}{x^{2}+\eta^{2}}=\frac{2 C \sqrt{-\rho}}{-4 \rho+\eta^{2}}=\frac{C \sqrt{-\xi-\frac{\eta^{2}}{4}}}{-2 \xi}
$$

valid inside the parabola $P_{0}: \xi>-\eta^{2} / 4$. A simple adjustment replaces $P_{0}$ by a larger parabola $P_{c}$ which includes the domain of interest. In particular, this estimate (which is really an estimate of the deviation of the solution from its upper bound along $P_{c}$ ) reaches its maximum along the $\xi$ axis, and is of order $1 / \sqrt{-\xi}$.

To obtain a bounded domain $\Omega$ we introduce a cut off boundary $\sigma=\left\{\xi=\xi^{*}\right\}$ in Section 3, with $\xi^{*}=\xi\left(\eta^{*}\right)$ given by (2.10) for a suitably large value of $\eta^{*}$. On $\sigma$ we impose a Dirichlet boundary condition $u=f(\eta)$. From the arguments in this section, we see that the estimate $f(\eta)=1+\mathcal{O}\left(1 / \sqrt{-\xi^{*}}\right)=1+\mathcal{O}\left(1 / \eta^{*}\right)$ is consistent with the asymptotics. We also set $f\left(\eta^{*}\right)=u^{*}=u\left(\eta^{*}\right)$ from the asymptotic condition (2.11) along the shock.

3. Preliminary Results. There are two natural ways to formulate the problem. Using symmetry, we can replace the domain in the upper half-plane by a domain consisting of the original problem plus its reflection in the line $\eta=0$. We truncate the infinite domain by introducing the curve $\sigma=\left\{\xi=\xi^{*}\right\}$, on which $u$ satisfies a Dirichlet boundary condition, $u=f$. The truncated domain has corners at $V$, where $\sigma$ meets $\Sigma$, and at the reflection of $V$, and at $\Xi_{a}$.

Alternatively, we can work in the upper half plane; then the boundary of $\Omega$ contains three different types of open sets. On $\sigma$, we have a Dirichlet condition; on $\Sigma$, there is the oblique derivative boundary condition, (2.9), corresponding to the free boundary problem; and on the symmetry boundary (the wall), $\eta=0, u$ satisfies the symmetry condition, $u_{\eta}=0$. Since the wall condition is also an oblique derivative condition, the problem is no more complicated when formulated this way. Denote the symmetry boundary by $\Sigma_{0}$, and the corner between $\sigma$ and $\Sigma_{0}$ by $W$.

In either formulation, the part of the boundary on which the oblique derivative boundary condition is imposed has two components, because there is no "normal derivative" at the corner $\Xi_{a}$. Lieberman's theory requires that $\Sigma$ be open, but not so for $\sigma$. Thus, the point $\Xi_{a}$ becomes a part of the Dirichlet boundary, at which we impose the condition $u=u_{R}$ or $u_{F}$ (if $a<a^{*}$ ) or $u_{F}$ (if $a>a^{*}$ ). We refer to the 

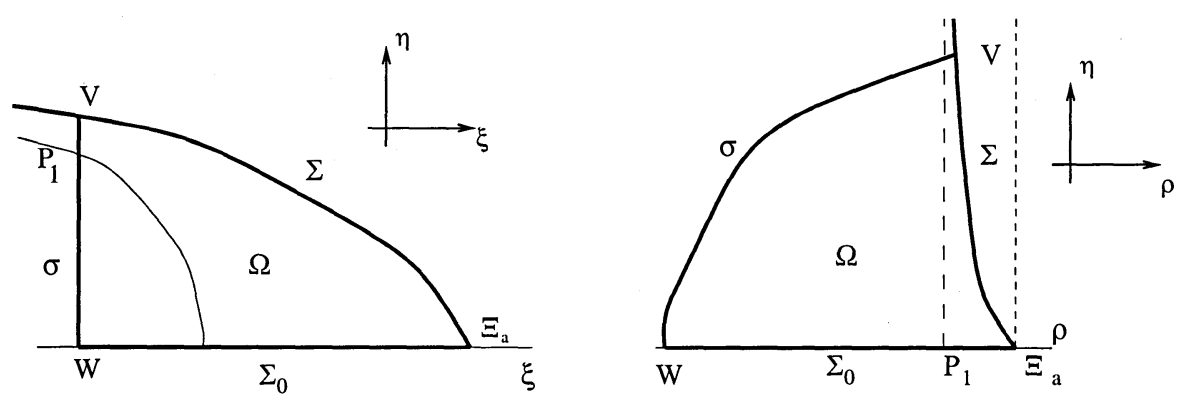

FIG. 3.1. Domains in the Two Coordinate Systems

value of $u$ at $\Xi_{a}$ as $u_{R}$ (for reflected) while recognizing that all we need is any subsonic condition.

The domain has three corners. Each has an opening angle which is bounded above and below. In Section 3.2, we introduce weighted Hölder spaces as in [8].

If we assume $\eta^{*}$ is large, then the coordinates of the point $V$ are given approximately by the asymptotic theory. In fact, we have, for $V=\left(\xi^{*}, \eta^{*}\right)$,

$$
\xi^{*}=-\frac{\left(\eta^{*}\right)^{2}}{4}+1+b\left(\eta^{*}\right)=-\frac{\left(\eta^{*}\right)^{2}}{4}+1+\frac{v_{0}}{\eta^{*}}+\frac{v_{1}}{\left(\eta^{*}\right)^{2}}+\frac{v_{2}}{\left(\eta^{*}\right)^{3}}+\frac{v_{3}-v_{0}^{2}}{\left(\eta^{*}\right)^{4}}+\ldots
$$

The value of $u$ at $V$ is given asymptotically:

$$
u^{*}=u(V)=1+\theta\left(\eta^{*}\right)=1+\frac{2 v_{0}}{\eta^{*}}+\frac{2 v_{1}}{\left(\eta^{*}\right)^{2}}+\frac{2 v_{2}}{\left(\eta^{*}\right)^{3}}+\frac{2 v_{3}-4 v_{0}^{2}}{\left(\eta^{*}\right)^{4}}+\ldots
$$

In the $(\rho, \eta)$ coordinate system, with $\rho=\xi+\eta^{2} / 4$, the parabola $P_{1}$ becomes the straight line $\rho=1$, to which the shock is asymptotic, and we have

$$
\rho^{*}=1+\frac{v_{0}}{\eta^{*}}+\frac{v_{1}}{\left(\eta^{*}\right)^{2}}+\frac{v_{2}}{\left(\eta^{*}\right)^{3}}+\frac{v_{3}-v_{0}^{2}}{\left(\eta^{*}\right)^{4}}+\ldots
$$

An estimate for the ellipticity ratio is found from the asymptotic formula

$$
u^{*}-\rho^{*}=\frac{v_{0}}{\eta^{*}}+\frac{v_{1}}{\left(\eta^{*}\right)^{2}}+\frac{v_{2}}{\left(\eta^{*}\right)^{3}}+\frac{v_{3}-3 v_{0}^{2}}{\left(\eta^{*}\right)^{4}}+\ldots
$$

so the value of $u-\rho$ at $V$ is bounded below by $v_{0} / 2 \eta^{*}$, say, if $\eta^{*}$ is large enough.

In $(\rho, \eta)$ coordinates, the cut off boundary $\sigma$ becomes the parabola $\rho=\xi^{*}+\eta^{2} / 4$ as in Figure 3.1. In solving the problem, we replace the free boundary by a fixed approximate shock position, and this also introduces an approximate cut off boundary $\sigma$ whose position changes with each approximation. Upper and lower bounds for $\sigma$ are stated in Section 3.1 and proved in Section 4.2.1.

3.1. Statement of the Problem. The proof of Theorem 1.1 about shock reflections reduces to solving a free boundary problem in the bounded domain $\Omega$.

The following theorem, which implies Theorem 1.1, is the main technical result of the paper. In stating this theorem, we refer to the geometry sketched in Figure 3.1 . The assumptions on the boundary value, $f$, on the cut off boundary are $f>1$, consistent with the asymptotic bounds of Section 2.4, and $u-\rho=f-\rho>0$ on $\sigma$, which ensures that the equation remains elliptic in $\Omega$. 
THEOREM 3.1. For $a \geq \sqrt{2}$ and $u_{R}$ a value giving a subsonic state at $\Xi_{a}$, and for any $f \in H_{1+\alpha}$ with $f>1$ and near unity and $f-\xi-\eta^{2} / 4>0$ on $\sigma$, the following problem has a solution in a finite neighborhood of $\Xi_{a}$ :

$$
\begin{aligned}
& \left.\begin{array}{rl}
(u-\xi) u_{\xi}-\eta u_{\eta}+v_{\eta} & =0 \\
-v_{\xi}+u_{\eta} & =0
\end{array}\right\} \text { in } \Omega, \\
& \left.\begin{array}{l}
\frac{d \xi}{d \eta}=-\frac{\eta}{2}-\sqrt{\xi+\frac{\eta^{2}}{4}-\frac{u+1}{2}} \\
\frac{d \xi}{d \eta}=-\frac{[v]}{[u]}
\end{array}\right\} \text { on } \Sigma \equiv\{\xi=\xi(\eta)\}, \\
& v=0 \text { on } \Sigma_{0} \\
& u=f \text { on } \sigma \text {, } \\
& u\left(\Xi_{a}\right)=u_{R}, \\
& \xi(0)=\xi_{a} \text {. }
\end{aligned}
$$

The functions $u$ and $v$ lie in Hölder spaces $H_{1+\alpha}^{(-\gamma)}$ for some values of $\gamma$ and $\alpha$ determined by $a$ and by $f$. The function $\xi$, representing the reflected shock, is in a Hölder space $H_{1+\alpha_{\Sigma}}$ for a value $\alpha_{\Sigma}$ also determined by a and by $f$.

The weighted Hölder spaces $H_{1+\alpha}^{(-\gamma)}$ are defined in Section 3.2.

We rephrase the boundary value problem as a second order equation for $u$ and reconstruct $v$ by integration, and we make three additional adaptations. First, we change coordinates to $(\rho, \eta)$. Second, we modify the operator $Q$ so that it is uniformly elliptic. We replace $Q(u)$ of $(2.3)$ with

$$
\widetilde{Q}(u)=\left(h(u-\rho) u_{\rho}+\frac{u}{2}\right)_{\rho}+u_{\eta \eta}=0,
$$

where

$$
h(x)= \begin{cases}x, & x \geq \varepsilon \\ \varepsilon, & x<\varepsilon\end{cases}
$$

and $\varepsilon$ is a small positive number which we specify in Section 5 . (Later, we will need $h^{\prime}$ in some constructions. It is straightforward to modify $h$ with a smoothing function in a small neighborhood (say, $\varepsilon^{2}$ ) of $\varepsilon$, so that $h^{\prime}$ is continuous and $0 \leq h^{\prime} \leq 1$. We assume this done, without further comment.)

Third, in addition to requiring a cut off function to ensure the ellipticity of $Q$, as implemented in $\widetilde{Q}$, we must ensure that the evolution equation for the shock position, (2.8), is well-defined; that is, we require $\rho-\bar{u} \geq 0$. Defining $g$ by

$$
g(x)=\left\{\begin{array}{ll}
x, & x \geq \delta * \\
\delta *, & x<\delta *
\end{array},\right.
$$

we modify the evolution operator for the shock in equation $(2.8)$ to $-\sqrt{g(\rho-\bar{u})}$.

Some differences between this problem and that solved in [8] are (1) the oblique derivative boundary condition is homogeneous, (2) we need a boundary condition at $\Xi_{a}$ (as the oblique derivative part of the boundary now has two components), and (3) there is no naturally occurring small parameter.

We first prove the following theorem for the modified problem. (The function $\beta$ was given in equation (2.9).) 
THEOREM 3.2. For $a \geq \sqrt{2}$ and $u_{R}$ a value giving a subsonic state at $\Xi_{a}$, for any $f \in H_{1+\alpha}$ with $f>1$ and near unity and $f-\rho>0$ on $\sigma$, and for positive values of $\varepsilon$ and $\delta *$, the following problem has a solution in $\Omega$ :

$$
\begin{gathered}
\widetilde{Q}(u) \equiv\left(h(u-\rho) u_{\rho}+\frac{u}{2}\right)_{\rho}+u_{\eta \eta}=0 \text { in } \Omega, \\
\frac{d \rho}{d \eta}=-\sqrt{g\left(\rho-\frac{u+1}{2}\right)} \text { on } \Sigma \equiv\{\rho=\rho(\eta)\} \\
N(u ; \rho) \equiv \beta \cdot \nabla u=0 \text { on } \Sigma \\
u_{\eta}=0 \text { on } \Sigma_{0} \\
u=f \text { on } \sigma \\
u\left(\Xi_{a}\right)=u_{R}, \\
\rho(0)=\xi_{a} \\
v(\rho, \eta)=\int_{0}^{\eta} \frac{y}{2} u_{y}-(u-\rho) u_{\rho} d y
\end{gathered}
$$

The functions $u$ and $v$ lie in Hölder spaces $H_{1+\alpha}^{(-\gamma)}$ for values of $\gamma$ and $\alpha$ determined by $a$ and by $f$. The function $\rho$, representing the reflected shock, is in a Hölder space $H_{1+\alpha_{\Sigma}}$ for a value $\alpha_{\Sigma}$ also determined by $a$ and by $f$.

Now, following the approach in [8], we solve the free boundary problem via a fixed boundary problem and a mapping, which we will show has a fixed point. The mapping is defined in three steps.

STEP 1

Define the problem. We begin with a function $\rho=\rho(\eta)$, defining an approximate boundary $\Sigma$. Here, $\rho$ belongs to a closed, convex subset $\mathcal{K}$ of the Banach space $H_{1+\alpha_{\Sigma}}$. The definition of $\mathcal{K}$ is

1. Smoothness: $\rho \in H_{1+\alpha_{\Sigma}}$ for a Hölder exponent which is specified in Section 4.2.1.

2. Initial Conditions: $\rho(0)=\xi_{a}$ and $\kappa_{\min } \leq \rho^{\prime}(0) \leq \kappa_{\max }$, where the bounds are chosen in Section 4.2 .

3. Monotonicity: $\rho^{\prime} \leq-\sqrt{\delta *}<0$; the value of $\delta *$ will also be specified in Section 4.2 .

4. Boundedness: $\rho_{L}(\eta) \leq \rho(\eta) \leq \rho_{R}(\eta)$. The definitions of $\rho_{L}$ and $\rho_{R}$ are given in Section 4.2.

The graph of $\rho(\eta)$ lies in a bounded set, $K$, in the $(\rho, \eta)$ plane.

Step 2

Solve the fixed boundary problem

$$
\begin{aligned}
& \widetilde{Q}(u) \equiv\left(h(u-\rho) u_{\rho}+\frac{u}{2}\right)_{\rho}+u_{\eta \eta}=0 \quad \text { in } \Omega, \\
& N(u)=0 \text { on } \Sigma \text {, } \\
& u_{\eta}=0 \ldots \text { on } \Sigma_{0} \text {, } \\
& u\left(\Xi_{a}\right)=u_{R},
\end{aligned}
$$

where $\sigma$ is the curve $\rho-\eta^{2} / 4=\rho\left(\eta^{*}\right)-\left(\eta^{*}\right)^{2} / 4$. This is a Lieberman-type mixed boundary value problem. The oblique derivative condition is posed on the two components $\Sigma$ and $\Sigma_{0}$, and Dirichlet conditions are posed on the complement $\sigma \cup \Xi_{a}$. One 
difference between this and the problem in [8] is that there are natural bounds on the solution. The domain $\Omega$ depends on the choice of $\rho(\eta)$ both through the boundary component $\Sigma$ and through $\sigma$. However, $\rho(\eta)$ is bounded above and below, so in Section 3.3 we obtain uniform estimates for $u$ in $\Omega$.

STEP 3

Update to $\tilde{\rho}$ the position of the boundary component $\Sigma$ by using the modification of the Rankine-Hugoniot condition (2.8), $\rho^{\prime}=-\sqrt{g(\rho-\bar{u})}$. We solve this as an ordinary differential equation for $\tilde{\rho}$, using the subsonic solution $u$ obtained in the previous step. Thus,

$$
\tilde{\rho}^{\prime}(\eta)=-\sqrt{g\left(\tilde{\rho}(\eta)-\frac{u(\rho(\eta), \eta)+1}{2}\right)} \text { with } \tilde{\rho}(0)=\xi_{a} .
$$

This defines a mapping $J$ on the set $\mathcal{K}$, by $\tilde{\rho}=J \rho$.

We prove Theorem 3.2 by means of a fixed point theorem, as in [8]. Then we deduce Theorem 3.1 by removing the cut off functions near $\Xi_{a}$ (Section 5), and citing Proposition 2.1 on the equivalence of the Rankine-Hugoniot conditions in Theorems 3.2 and 3.1. We get a local result only, in Theorem 3.1, because we cannot, at this point, eliminate the cut off functions everywhere.

Finally, Theorem 1.1 is a restatement of Theorem 3.1 which includes the (selfevident) supersonic part of the flow.

3.2. Hölder Norms Used in this Paper. Let $X=(\rho, \eta)$; let $D=\left(D_{1}, D_{2}\right)$ denote partial derivatives, and $D^{k} u$ the set of $k$-th order derivatives. The definitions in this section follow Gilbarg and Trudinger, [13], and Lieberman, [15, 16].

For functions defined on an open set $S$ in $\mathbf{R}^{2}$, the supremum norm and Hölder semi-norms are

$$
|u|_{0 ; S}=\sup _{X \in S}|u(X)| \text { and }[u]_{\alpha ; S}=\sup _{X, Y \in S} \frac{|u(X)-u(Y)|}{|X-Y|^{\alpha}}
$$

for $0<\alpha \leq 1$. Hölder norms of any order are

$$
|u|_{\alpha ; S}=|u|_{0 ; S}+[u]_{\alpha ; S} \text { for } \quad 0<\alpha \leq 1
$$

and, for $a=k+\alpha$ where $k$ is an integer and $0<\alpha \leq 1$,

$$
|u|_{a ; S}=\sum_{j<k}\left|D^{j} u\right|_{0 ; S}+\left|D^{k} u\right|_{\alpha ; S}
$$

The space of functions whose $(k+\alpha)$-Hölder norm is finite is denoted $H_{k+\alpha}$. For $\Sigma=\left\{(\rho(\eta), \eta) \mid 0<\eta<\eta^{*}\right\}$, we say $\Sigma \in H_{1+\alpha}$ if $\rho \in H_{1+\alpha}\left(\left(0, \eta^{*}\right)\right) ;|\Sigma|_{1+\alpha}$ denotes the $H_{1+\alpha}$ norm of $\xi$.

We let $\mathbf{V}=\left\{V, W, \Xi_{a}\right\}$ denote the set of corners of $\Omega$, and let

$$
d_{\mathbf{V}}(X) \equiv \min \left\{|X-V|,|X-W|,\left|X-\Xi_{a}\right|\right\}
$$

be the distance from a point $X \in \Omega$ to the corners.

Weighted or partially interior seminorms are defined as follows. For a subset $S$ of $\partial \Omega$, define

$$
\Omega_{\delta: S}=\{X \in \Omega \mid \operatorname{dist}(X, S)>\delta\}
$$


For any $a>0$ and $a+b \geq 0$, the weighted norms are ( $S^{c}$ is the complement of $S$ in $\partial \Omega)$

$$
|u|_{a ; \Omega \cup S^{c}}^{(b)}=\sup _{\delta>0} \delta^{a+b}|u|_{a ; \Omega_{\delta ; S}}
$$

The set of functions on $\Omega$ with finite norm $|u|_{a ; \Omega \cup S^{c}}^{(b)}$ is denoted $H_{a ; \Omega \cup S^{c}}^{(b)}$. As in [8], we let $S^{c}=\Sigma \cup \Sigma_{0} \cup \sigma=\partial \Omega-\mathbf{V}$ and we define $H_{a}^{(b)}=H_{a ; \Omega \cup \Sigma \cup \Sigma_{0} \cup \sigma}^{(b)}$.

These spaces have a compactness property, [12, 14]: for $0<b^{\prime}<b, 0<a^{\prime}<a$, $a \geq b$ and $a^{\prime} \geq b^{\prime}$, a bounded sequence in $H_{a}^{(-b)}$ is precompact in $H_{a^{\prime}}^{\left(-b^{\prime}\right)}$.

3.3. Bounds on the Solution. Because the boundary conditions on $\Sigma$ and $\Sigma_{0}$ are homogeneous (unlike the situation in [8]), we can bound solutions of (3.3) above and below. Let

$$
m=\min _{\eta}\{f(\eta)\} \quad M=\max _{\eta}\left\{f(\eta), u_{R}\right\}=u_{R},
$$

and suppose that $m>1$.

Proposition 3.3. Any differentiable solution $u$ of the fixed boundary problem (3.3) satisfies $m<u<M$ in $\Omega$.

Proof. The operator is uniformly elliptic, and by the maximum principle, the extrema of $u$ are achieved on $\partial \Omega$. Suppose there is an extremum at a point $\Xi_{0}$ on $\Sigma$ or $\Sigma_{0}$. Then the tangential derivative along the boundary satisfies $u^{\prime}\left(\Xi_{0}\right)=0$, since this value is also an extremum of the function restricted to the boundary. This, combined with $\beta \cdot \nabla u=0$, implies that $\nabla u\left(\Xi_{0}\right)=0$. However, this violates the Hopf lemma, (a version of the strong maximum principle), as stated, for example, in Lemma 3.4 of Gilbarg and Trudinger, [13]. Hence, the extrema are attained on $\sigma$ or at $\Xi_{a}$. If $f$ is close to unity, the maximum is $u_{R}$ at $\Xi_{a}$ and the minimum is attained on $\sigma$.

We study the lower bound of $w=u-\rho$.

Proposition 3.4. The function $w=u-\rho$ attains its minimum on $\sigma$ or $\Sigma$.

Proof. The function $w$ satisfies the equation

$$
h(w) w_{\rho \rho}+w_{\eta \eta}+h^{\prime} w_{\rho}^{2}+\left(h^{\prime}+\frac{1}{2}\right) w_{\rho}+\frac{1}{2}=0 .
$$

Noting the sign of the constant term, we use Theorem 3.5 of [13] to show that the minimum of $w$ occurs on $\partial \Omega$; as in Proposition 3.3 , we can rule out $\Sigma_{0}$ since $w_{\eta}=$ $u_{\eta}=0$ there.

In fact, $w$ remains positive on $\Sigma$.

Proposition 3.5. The function $w$ cannot obtain a non-positive minimum on $\Sigma$.

Proof. Suppose $w$ attains a non-positive minimum on $\Sigma$, say at $X_{0}$. Then by the Hopf lemma (Lemma 3.4 in [13]), we have

$$
\frac{\partial w\left(X_{0}\right)}{\partial \nu}<0
$$


where $\nu$ is an outward normal. We rewrite (3.5), using $\nu=\left(1,-\rho^{\prime}\right)$, as

$$
\frac{\partial w\left(X_{0}\right)}{\partial \nu}=\left(u_{\rho}-1\right)-\rho^{\prime} u_{\eta}<0
$$

and $X_{0}$ is an extremum so

$$
w^{\prime}\left(X_{0}\right)=\rho^{\prime}\left(u_{\rho}-1\right)+u_{\eta}=0 .
$$

Solving (3.7) for $u_{\eta}$ and using the result in (3.6) we get

$$
0>\left(u_{\rho}-1\right)-\rho^{\prime} u_{\eta}=\left(u_{\rho}-1\right)\left(1+\left(\rho^{\prime}\right)^{2}\right),
$$

which implies that

$$
u_{\rho}\left(X_{0}\right)<1
$$

Now consider the oblique derivative boundary condition, (2.9). Using (3.7), we can write the boundary condition at $X_{0}$ as

$$
0=u_{\rho} \rho^{\prime}\left(\frac{2 u-2}{8}\right)+\rho^{\prime}\left(\frac{5 u+3}{8}-\rho\right)
$$

Now by using inequality (3.8), and the bounds $\rho^{\prime}<0$ and $u>1$ on $\Sigma$ (from Proposition 3.3, and assuming $m>1$ ), equation (3.9) implies that at $X_{0}$

$$
0>\rho^{\prime}\left(\frac{2 u-2}{8}\right)+\rho^{\prime}\left(\frac{5 u+3}{8}-\rho\right) \text {. }
$$

Since $w=u-\rho \leq 0$ at $X_{0}$, we let $\mu \geq 0$ be the constant defined by

$$
u\left(X_{0}\right)=\rho-\mu .
$$

Using (3.11), we can write the inequality (3.10) as

$$
0>\rho^{\prime}\left(\frac{2(\rho-\mu)-2}{8}\right)+\rho^{\prime}\left(\frac{5(\rho-\mu)+3}{8}-\rho\right)=\rho^{\prime}\left(\frac{-\rho+1}{8}-\frac{7 \mu}{8}\right) .
$$

But since $\rho^{\prime}<0$, this means the second factor is nonnegative, and since $\rho>1$ on $\Sigma$, we obtain a contradiction. We see that $\mu$ is positive and is bounded below by $(\rho-1) / 7$. Therefore $w>0$ on $\Sigma$.

In Section 5 we use Proposition 3.5 to eliminate the cut off function $h$ and replace $\widetilde{Q}$ by $Q$.

4. Proof of the Main Theorem. There are two parts to the proof of Theorem 3.2: a demonstration that each fixed boundary problem has a solution for any $\rho$ in $\mathcal{K}$, and then, using estimates we derive in solving the fixed problem, that the mapping $J$ has a fixed point.

4.1. The Fixed Boundary Problem. We carry out Step 2 as outlined in Section 3.1, solving the fixed boundary problem (3.3). Throughout, $\Omega$ is a domain whose boundary is the curvilinear triangle with sides $\sigma=\left\{\rho-\eta^{2} / 4=\rho\left(\eta^{*}\right)-\left(\eta^{*}\right)^{2} / 4\right\}$, $\Sigma=\{\rho=\rho(\eta)\}$ and $\Sigma_{0}=\{\eta=0\}$. Here, $\eta^{*}$ is a fixed cutoff value. The main result is the following theorem. 
THEOREM 4.1. Let $\Omega$ be the Lipschitz domain described above, with $\Sigma \in H_{1+\alpha_{\Sigma}}$ for some $0<\alpha_{\Sigma}<1$. Then for some $\gamma<1$ there exists a solution $u$ of the problem (3.3). The solution is in $H_{1+\alpha_{*}}^{(-\gamma)}$ for all $\alpha_{*}$ with $0<\alpha_{*} \leq \alpha_{\Sigma}$.

Both boundary components $\Sigma$ and $\sigma$ of $\Omega$ depend on the function $\rho$ defining $\Sigma$. However, the boundedness of $K$, the set containing the graph of $\rho$, gives uniform bounds on the diameter of $\Omega$ and on the contribution of the domain to the ellipticity and obliqueness constants. The dependence of most of our Hölder estimates on the boundedness of $\Omega$ is the main reason we cannot extend our result to unbounded domains. In the remainder of the paper, so as not to burden the proofs with repetitive detail, we do not refer to this dependence. The bounds on $\rho$ and on $\sigma$ are stated in Section 3.1, STEPS 1 and 2, and proved in Section 4.2.1. Because of the bounds on $u$ and $\rho$, and on $\Omega$, the operators $\widetilde{Q}$ and $N$ are uniformly elliptic and uniformly oblique, respectively. In addition, $\Omega$ satisfies the exterior cone condition [13, page 203].

To prove Theorem 4.1, we first solve a linearized problem, and then apply a fixed point theorem.

4.1.1. The Linear Problem. Lieberman's theorem in [15] for the linear problem requires $\Sigma \in H_{2+\alpha}$. Since the boundary component $\Sigma$ lacks this smoothness, we solve the linear problem as a limit of problems on regularized domains. The bounds we obtain are uniform in the smoothed domains.

Define linear operators

$$
\begin{aligned}
& L u \equiv D_{i}\left(a^{i j}(X) D_{j} u\right)=\left(h(z-\rho) u_{\rho}+\frac{u}{2}\right)_{\rho}+u_{\eta \eta} \text { in } \Omega \text {, } \\
& M u \equiv \beta^{i}(X) D_{i} u \quad=\beta^{1}(z) u_{\rho}+\beta^{2}(z) u_{\eta} \text { on } \Sigma \text {, }
\end{aligned}
$$

where

$$
z \in H_{1+\epsilon}^{\left(-\gamma_{1}\right)}
$$

for some $\gamma_{1}$ with $0<\gamma_{1} \leq \alpha_{\Sigma}$, and $\epsilon$ is a Hölder exponent to be chosen later. For convenience, we replace $u$ by $u-u_{R}$ in the linear problem, denoting the new function by $\tilde{u}$. Then the linear problem is

$$
\begin{array}{r}
L \widetilde{u}=0 \text { in } \Omega, \quad M \widetilde{u}=0 \text { on } \Sigma, \quad \widetilde{u}_{\eta}=0 \text { on } \Sigma_{0}, \\
\quad \widetilde{u}=f-u_{R} \text { on } \sigma, \quad \widetilde{u}\left(\Xi_{a}\right)=0 .
\end{array}
$$

The key point, as in [8], is that the weighted Hölder space in which we find $\tilde{u}$ does not depend on the corresponding Hölder exponent $\gamma_{1}$ of the coefficient $z$. The weighted Hölder bounds for $\widetilde{u}$ involve the growth of $\widetilde{u}$ at the corners (the vertex set V). Since the corner bounds are local, we replace $\widetilde{u}$ there by a function $\widetilde{u}_{1}$ defined near each vertex point $V_{i}$ as $\widetilde{u}-\widetilde{u}\left(V_{i}\right)$. Then we can establish existence and the Hölder bounds of the following theorem.

THEOREM 4.2. Let $\Sigma$ be in $H_{1+\alpha_{\Sigma}}$ and $z$ be in $H_{1+\epsilon}^{\left(-\gamma_{1}\right)}$ for exponents $0<\epsilon \leq$ $\alpha_{\Sigma}<1$ and $0<\gamma_{1}<1$. Suppose also that $z \geq m>1$ and that for some constant $\bar{m}$,

$$
\left|D\left(a^{i j}\right)\right| \leq \bar{m} d_{\mathbf{V}}^{\gamma_{1}-1}
$$

and that $f \in H_{1+\alpha}$ is bounded and $f \geq m>1$. Then there exists a unique solution $\tilde{u} \in H_{1+\alpha_{\Sigma}}^{(-\gamma)}$ of the linear problem (4.2), where $\gamma$ is determined by the geometry of $\Omega$ 
and by the operators $L$ and $M$. The solution $\widetilde{u}$ satisfies the two estimates

$$
\begin{aligned}
& |\widetilde{u}|_{1+\alpha_{\Sigma}}^{(-\gamma)} \leq C\left(\left|f-u_{R}\right|_{\gamma}+\sup \left|d_{\mathbf{V}}^{-\gamma} \widetilde{u}_{1}\right|\right), \\
& |\widetilde{u}|_{1+\alpha_{\Sigma}}^{(-\gamma)} \leq C_{1}\left(\left|f-u_{R}\right|_{\gamma}+|\widetilde{u}|_{0}\right),
\end{aligned}
$$

where $C$ and $C_{1}$ are positive constants which depend on $\epsilon,[z]_{\alpha_{\Sigma}},[\beta(z)]_{\alpha_{\Sigma}}$, and $|\Sigma|_{1+\alpha_{\Sigma}}$, and $C_{1}$ also depends on $\bar{m}$.

Proof. We follow the technique in [8]. It is convenient to divide the proof into the same four parts.

\section{PART 1}

We first obtain an a priori $L^{\infty}$ estimate for a solution $\widetilde{u}$. The estimate in Proposition 3.3 holds also for the linear problem, and so $|\widetilde{u}|_{0} \leq\left|f-u_{R}\right|_{0}$.

\section{PART 2}

Now we prove that any solution of (4.2) satisfies estimate (4.4). As explained in the preamble to the Theorem, this estimate is exactly like that in Theorem 3.2 of [8], with the two changes that now the Dirichlet boundary condition is nonhomogeneous and contributes the term $\left|f-u_{R}\right|_{\gamma}$ as in [13, page 139], and that we subtract an affine function from $\tilde{u}$ to obtain the growth rates at the corners. At each corner, this then follows exactly the proof in [8].

To get (4.5), we now observe that $\left|d_{\mathbf{V}}^{-\gamma} \widetilde{u}_{1}\right| \leq\left|\widetilde{u}_{1}\right|_{\gamma}$, and that $|\widetilde{u}|_{\gamma} \leq C|\widetilde{u}|_{1+\alpha}^{(-\gamma)}$. Finally, we quote Theorem 1 from [16] with $f_{1}=f_{2}=0$ and $f_{3}=f-u_{R}$, to replace $|\widetilde{u}|_{1+\alpha}^{(-\gamma)}$ by $\left|f-u_{R}\right|_{\gamma}+|\widetilde{u}|_{0}$. Because of the a priori bound on $\widetilde{u}$, the term $|\widetilde{u}|_{0}$ is not needed in (4.5).

\section{$\underline{\text { PART } 3}$}

We approximate $\Omega$ by a sequence of domains $\Omega_{k}$ using a sequence of smooth curves $\Sigma_{k}$ in $\Omega$ which approximate $\Sigma$. The hypotheses of Theorem 1 of [15] are satisfied, so we obtain existence of a solution $\widetilde{u}_{k}$ in $\Omega_{k}$ for each $k$. The set $\mathbf{V}$ in this theorem is the set of all three vertices, while the set $\sigma$ in that theorem is the same as the set $\sigma$ in this paper (that is, it does not include the point $\Xi_{a}$ ), and the $\Sigma$-wedge and exterior cone conditions are satisfied. The solutions satisfy (4.4) and (4.5) for each $u_{k}$, with constants which do not depend on $k$.

\section{PART 4}

The argument is identical to that in [8]: As $k \rightarrow \infty, \Omega_{k} \rightarrow \Omega$. Since (4.4) holds for each $k$, the sequence $\widetilde{u}_{k}$ is uniformly bounded in $H_{1+\alpha}^{(-\gamma)}$. By the Arzela-Ascoli theorem there exists a convergent subsequence which converges uniformly to $\widetilde{u}$, a weak solution of (4.2). Uniqueness follows from the linear version of Proposition 3.3. This completes the proof of Theorem 4.2.

Although the construction gives us only a weak solution, we know, since $z \in$ $H_{1+\epsilon}^{\left(-\gamma_{1}\right)}$, that $\tilde{u} \in C^{2}(\Omega)([13$, Theorem 6.2]).

4.1.2. The Nonlinear Problem. Now we solve the nonlinear fixed boundary problem, (3.3), by using a fixed point theorem:

TheOREM 4.3 (ThEOREM 11.3 of [13]). Let $T$ be a compact mapping of a Banach space $\mathcal{B}$ into itself, and suppose that there exists a constant $M$ such that

$$
\|u\|_{\mathcal{B}} \leq M
$$

for all $u \in \mathcal{B}$ and $\tau \in[0,1]$ satisfying $u=\tau T u$. Then $T$ has a fixed point.

The procedure is very similar to that in [8]. We define $T: H_{1+\epsilon}^{\left(-\gamma_{1}\right)} \rightarrow H_{1+\epsilon}^{\left(-\gamma_{1}\right)}$ by letting $u=T z$ be the unique weak solution, $u=\widetilde{u}+u_{R}$, of the linear mixed boundary 
problem (4.2) which we have just constructed. By Theorem 4.2, $T\left(H_{1+\epsilon}^{\left(-\gamma_{1}\right)}\right) \subset H_{1+\alpha_{\Sigma}}^{(-\gamma)}$, so the operator $T$ is compact if $\gamma_{1}<\gamma$ and $\epsilon<\alpha_{\Sigma}[12,14]$. At this point, we may take $\epsilon=\alpha_{\Sigma} / 2$, and $\gamma_{1}=\gamma / 2$.

Throughout, $\alpha_{\Sigma}$ denotes the smoothness of the boundary $\Sigma$, fixed in this section, and also denotes the smoothness of the coefficients in the oblique derivative boundary condition (the function $\beta$ in (2.9)). We have a geometric upper bound for $\gamma$, but we do not specify $\alpha_{\Sigma}$ until we consider the free boundary problem in the next subsection.

We need to show that there exists an $M>0$ such that

$$
\|\widetilde{u}\|_{\mathcal{B}} \leq M
$$

for all functions $\widetilde{u} \in \mathcal{B} \equiv H_{1+\alpha_{\Sigma} / 2}^{\left(-\gamma_{1}\right)}$, which solve $\widetilde{u}=\tau T \widetilde{u}$, or

$$
\begin{aligned}
& \widetilde{Q}(\widetilde{u}) \equiv\left(h(u-\rho) \widetilde{u}_{\rho}+\frac{\widetilde{u}}{2}\right)_{\rho}+\widetilde{u}_{\eta \eta}=0 \quad \text { in } \Omega \text {, } \\
& N(\widetilde{u}) \equiv \quad \beta^{1}(u) \widetilde{u}_{\rho}+\beta^{2}(u) \widetilde{u}_{\eta}=0 \quad \text { on } \Sigma \text {, } \\
& \widetilde{u}_{\eta}=0 \quad \text { on } \Sigma_{0}, \\
& \widetilde{u}=\tau\left(f-u_{R}\right) \text { on } \sigma, \\
& \widetilde{u}\left(\Xi_{a}\right)=0 \text {. }
\end{aligned}
$$

(We have again denoted $u-u_{R}$ by $\widetilde{u}$.) If $\widetilde{Q}(\widetilde{u})=0$ has a solution in $H_{1+\alpha}^{(-\gamma)}$, then $\widetilde{u} \in$ $C^{2}(\Omega)$, because we can look at $\widetilde{u}$ as the solution of a linear problem whose coefficients have sufficient smoothness, as in the comment following the proof of Theorem 4.2.

The key estimate is the following.

Lemma 4.4. Suppose that $\widetilde{u}$ is a solution of (4.6). Then there exist constants $\alpha_{*}>0$ and $M>0$ such that

$$
|\widetilde{u}|_{1+\alpha_{*}}^{(-\gamma)} \leq M, \quad \forall \tau \in[0,1] .
$$

Proof. We proceed exactly as in the proof of Lemma 3.7 of [8].

The first step is an $L^{\infty}$ bound for $u$; this is a consequence of the maximum principle, Proposition 3.3, as before.

Next, we need an estimate of $\left|d_{\mathbf{V}}^{-\gamma} u\right|$ which is independent of $z$. For this estimate, we use linear operators that give upper and lower solutions. We define

$$
\bar{L} v=h(u-\rho) v_{\rho \rho}+\left(\frac{1}{2}-h^{\prime}\right) v_{\rho}+v_{\eta \eta}, \quad \bar{M} v=\beta(u) \cdot \nabla v .
$$

Since $\bar{L}$ differs from $\widetilde{Q}$ by the quantity $h^{\prime} u_{\rho}^{2} \geq 0$, we know that $\widetilde{Q}(\widetilde{u})=0$ implies $\bar{L} \widetilde{u}=\widetilde{Q}(\widetilde{u})-h^{\prime} \widetilde{u}_{\rho}^{2} \leq 0$; thus $\widetilde{u}$ is a supersolution of the linear problem

$$
\begin{aligned}
\bar{L} v=0 \text { in } \Omega, \quad \bar{M} v=0 \text { on } \Sigma, \quad v_{\eta}=0 \text { on } \Sigma_{0}, \\
v=\tau\left(f-u_{R}\right) \text { on } \sigma, \quad v\left(\Xi_{a}\right)=0 ;
\end{aligned}
$$

that is, $\widetilde{u} \geq v$, and $v$ gives a lower bound for $\widetilde{u}$. We get an upper bound from $v_{\text {sub }}=\left(e^{k \widetilde{u}}-1\right) / k$ for sufficiently large $k$, since with $v=v_{\text {sub }}$ we have

$$
\bar{L} v=e^{k \widetilde{u}}\left(-h^{\prime} \widetilde{u}_{\rho}^{2}+k\left(h \widetilde{u}_{\rho}^{2}+\widetilde{u}_{\eta}^{2}\right)\right)>0
$$


if $k>h^{\prime} / h$. Thus $v_{\text {sub }}$ is a subsolution of the problem

$$
\begin{aligned}
\bar{L} W=0 \text { in } \Omega, \bar{M} W=0 \text { on } \Sigma, W_{\eta} & =0 \text { on } \Sigma_{0}, \\
W & =\frac{1}{k}\left(e^{\tau\left(f-u_{R}\right)}-1\right) \text { on } \sigma, W\left(\Xi_{a}\right)=0 ;
\end{aligned}
$$

that is $v_{\text {sub }} \leq W$. However, since $v_{\text {sub }}=\widetilde{u}+k \widetilde{u}^{2} / 2+\ldots>\widetilde{u}$, we see that the solution $W$ of the linear problem (4.9) gives an upper bound for $\widetilde{u}$. As in [8], solutions of the linear problems (4.8) and (4.9) give corner barriers and we get

$$
\left|d_{\mathbf{V}}^{-\gamma} u\right| \leq C
$$

We need to improve these estimates to $|u|_{1+\alpha_{*}}^{(-\gamma)} \leq M$. This proceeds exactly as in [8]; as in that paper, the nonlinearity is quadratic, and a bootstrapping argument gives $\alpha_{*}=\min \left\{\gamma, \alpha_{\Sigma}\right\}$.

This lemma and the fixed point theorem now give existence of solutions to the nonlinear fixed boundary problem, and complete the proof of Theorem 4.1.

4.2. The Free Boundary Problem. We now complete Step 3 of the program outlined in Section 3.1. The two points to verify are that $J$ maps $\mathcal{K}$ into itself and that $J$ is a precompact mapping. Then we apply a corollary of the Schauder fixed point theorem:

TheOREM 4.5 (COROLlary 11.2 of [13]). Let $\mathcal{K}$ be a closed, convex subset of a Banach space $\mathcal{B}$ and let $T$ be a continuous mapping from $\mathcal{K}$ into itself such that the image $T \mathcal{K}$ is precompact. Then $T$ has a fixed point.

4.2.1. Bounds on the Mapping $J$. As in [8], we have used uniform ellipticity and uniform obliqueness to find a solution $u$ to the fixed boundary problem, in an appropriate weighted Hölder space. There are some minor differences with [8], because the bounds on $u$ are not controlled by a function $\psi$ determined by an upstream boundary condition, as they were in that paper; instead, in the present work we use the a priori bounds for $u$ given by Proposition 3.3.

However, checking that $J$ maps $\mathcal{K}$ to itself requires a different argument, since we are not perturbing about a constant. In this section, we show that because we are solving a differential equation in (3.4), the smoothness condition, item 1 , in the definition of $\mathcal{K}$ in Section 3.1, holds with an increase in regularity (giving the compactness we require). Also, the initial conditions, item 2 in the definition of $\mathcal{K}$, are preserved: The condition $\tilde{\rho}(0)=\xi_{a}$ is part of the definition of $\tilde{\rho}$, and $\tilde{\rho}^{\prime}(0)$ has the exact value $\kappa_{R}$ because $u\left(\Xi_{a}\right)=u_{R}$; we choose the bounds in item 2 so that $\kappa_{\min }<\kappa_{R}<\kappa_{\max }$. Furthermore, monotonicity of $\tilde{\rho}$, item 3 , follows since $\tilde{\rho}^{\prime}$ is strictly negative. In addition, we require that

(a) $\tilde{\rho}$ be defined for all $\eta \in\left[0, \eta^{*}\right]$. This follows since we have modified the integrand so it is always positive, by using the cut off function $g$ defined in $(3.2)$; and

(b) $\tilde{\rho}$ be bounded, as in item 4 , between $\rho_{L}$ and $\rho_{R}$. This is achieved by the choice of the value of $\delta *$ in (3.2), as we demonstrate following Proposition 4.6 .

Thus, bounds are effected by introducing the cut off function $g$ and by choosing a large physical region $K$ in which the graph of $\rho$ is permitted to lie. It is, in part, because of this rather crude approximation to the domain of the shock that we obtain only a local result. 
In selecting the cut off parameters, we first choose $\eta^{*}$. For example, we could take $\eta^{*}$ to be large enough that the asymptotic series of Sections 2.3 and 2.4 give estimates by multiples of their first terms. These series are defined in terms of a set of coefficients for the asymptotic values of the function $v(\eta)$ along the shock, which we will assume given and fixed. In principle, $v$ is part of the solution, and the problem is not complete until we have proved that these coefficients are bounded. As stated in the Introduction, our result is still incomplete in this respect. Thus, for the purposes of this convergence argument, we fix $\eta^{*}$. In fact, as we do not use the asymptotic values in the definition of $\delta *$, there is no lower bound on $\eta^{*}$.

Next, we choose the boundary condition $u=f$ on $\sigma$ so that $f$ is bounded below by a constant $m>1$ on $\sigma$. As indicated in Section 2.4, we expect $u-1=\mathcal{O}\left(1 / \eta^{*}\right)$ on $\sigma$. However, it is not necessary to specify this bound precisely, as long as $\min f \geq m>1$.

Finally, we specify $\delta *$ in terms of $m$ and $\eta^{*}$.

Proposition 4.6. If the cut off in equation (3.2) is chosen with

$$
\delta *<\left(\frac{m-1}{2 \eta^{*}}\right)^{2}
$$

then $\tilde{\rho}$ is a monotonic curve with $\tilde{\rho}(\eta)>1$ for all $\eta \in\left[0, \eta^{*}\right]$.

Proof. At all values $\eta$ where $\tilde{\rho}-\bar{u} \geq \delta *>0$, we have $\tilde{\rho}-1 \geq(m-1) / 2$. There is at least one value of $\eta$ where this holds, namely $\eta=0$. Furthermore, the change in $\tilde{\rho}$, over values of $\eta$ where the inequality $\tilde{\rho}-\bar{u} \geq \delta *$ does not hold, is $\sqrt{\delta *}$ per unit interval in $\eta$, and thus the total change is bounded by $\eta^{*} \sqrt{\delta *}$. But if the inequality (4.10) holds, then this total change is less than $(m-1) / 2$, and hence $\tilde{\rho}$ is bounded below by a number $\rho_{\text {min }}>1$.

From the proof, we see that $\tilde{\rho}-1$ is bounded below by a positive number, consistent with the asymptotic behavior of the actual free boundary.

We now define $\rho_{L}$ and $\rho_{R}$ to complete item 4 in the definition of the set $\mathcal{K}$. A lower bound for $\tilde{\rho}^{\prime}$ is $-\sqrt{\max (\tilde{\rho}-\bar{u})}$, and

$$
\max (\tilde{\rho}-\bar{u}) \leq \max \tilde{\rho}-\min \bar{u} \leq \xi_{a}-1
$$

by integrating this we obtain

$$
\rho_{L}= \begin{cases}\xi_{a}-\eta \sqrt{\xi_{a}-1}, & \eta \leq \eta_{c} \\ 1+\sqrt{\delta *\left(\eta^{*}-\eta\right),} & \eta>\eta_{c}\end{cases}
$$

where $\eta_{c}$ is the value of $\eta$ at which the two straight line bounds intersect. On the other hand,

$$
\tilde{\rho}^{\prime} \leq-\sqrt{\delta *}
$$

Thus,

$$
\rho_{R}=\xi_{a}-\eta \sqrt{\delta *}
$$

provides an upper bound for the functions in $\mathcal{K}$. Finally, we choose

$$
\kappa_{\min }=-\sqrt{\xi_{a}-1}, \quad \kappa_{\max }=-\sqrt{\delta *}
$$

in item 2 in the definition of $\mathcal{K}$. We have now proved 
Proposition 4.7. If $\delta *$ satisfies inequality (4.10), then $J(\mathcal{K}) \subset \mathcal{K}$.

Note that as a consequence of these bounds, we obtain upper and lower bounds for the curve $\sigma$ and thus for the diameter of $\Omega$, as needed for a number of the Hölder estimates.

We also require compactness of the mapping $J$. Let $\alpha_{\Sigma}$ be the Hölder exponent of the set $\mathcal{K}$. Then compactness results from the choice of $\alpha_{\Sigma}$, as in [8].

Proposition 4.8. The set $J \mathcal{K}$ is precompact in $H_{1+\alpha_{\Sigma}}$ for sufficiently small $\alpha_{\Sigma}$.

Proof. The quasilinear elliptic operator and the boundary conditions are similar to the problem considered in [8]. Hence we obtain a bound on the right hand side of (3.4), $|\sqrt{g(\tilde{\rho}-\bar{u})}|_{\alpha_{1}} \leq C$, as in Proposition 4.2 of [8], for any $\alpha_{1} \leq \gamma$. The existence and uniqueness theorem for ordinary differential equations now gives $|\tilde{\rho}|_{1+\gamma} \leq C \eta^{*}$. We complete the proof by choosing $\alpha_{\Sigma}=\gamma / 2$.

To use Theorem 4.5, we also need the continuity of $J$.

\section{Proposition 4.9. The mapping $J$ is continuous.}

Proof. Since the mapping is compact, continuity is straightforward. In this case, we need to show that $\left\|J \rho_{1}-J \rho_{2}\right\|_{1+\alpha} \rightarrow 0$ if $\left\|\rho_{1}-\rho_{2}\right\|_{1+\alpha} \rightarrow 0$. Construction of $\tilde{\rho}$ from $\rho$ takes place in two stages. The dependence of the integral is clearly continuous in $\rho$; the dependence of $u$ on $\rho$ can be established by finding a priori bounds, in $H_{1+\alpha}^{(-\gamma)}$, for $z=u\left(\rho_{1}\right)-u\left(\rho_{2}\right)$ in the domain between the two curves $\rho_{1}$ and $\rho_{2}$. As those bounds shrink with the domain, we obtain continuity.

Propositions 4.7, 4.8 and 4.9 together verify the hypotheses of Theorem 4.5, and we conclude the existence of a fixed point:

LEMMA 4.10. The operator $J$ has a fixed point $\rho \in H_{1+\alpha_{\Sigma}}$.

From the fixed point $\rho$ we use Theorem 4.1 to obtain the solution $u$ in $H_{1+\alpha *}^{(-\gamma)}$ for any $\alpha * \leq \alpha_{\Sigma}$. The function $v$ is recovered by integration, and is in the same space. This completes the proof of Theorem 3.2.

5. Conclusions: Removal of the Cut Off Functions. Theorem 3.2 concerns the solution to a modified problem. In order to complete the proof of Theorem 3.1, we must replace the cut off functions $g$ and $h$ of Section 3.1 with identity functions. In the case of $g$, the modification of the shock evolution operator defined in equation (3.2), it is clear that $g$ is the identity unless $u \geq 2 \rho-1-\delta *$, where $\delta *$ is small. Now, $u \leq u_{R}$ by the maximum principle (Proposition 3.3), so this cut off will not apply until $\rho \leq\left(u_{R}+1+\delta *\right) / 2$, a quantity less than $\xi_{a}$.

The other cut off function, $h$, defined in equation (3.1), comes into play if $u \leq$ $\rho+\varepsilon$. Recall that, in Theorem 3.1, $f$ was chosen so that $w>0$ on $\sigma$. In the proof of Proposition 3.5, we saw that $w=u-\rho$ is bounded below on $\Sigma$ by a quantity $\mu \geq \min _{\Sigma}(\rho-1) / 7$. Now, Proposition 4.6 showed that $\tilde{\rho}>1$. Furthermore, in the proof of Proposition 4.6, one can estimate the lower bound of $\tilde{\rho}-1$ by a constant depending only on $\delta *, m$, and $\eta^{*}$. Hence we can bound the set, $K$, containing the graph of $\rho$, by a constant greater than unity, and thus obtain a lower bound for $\mu$ which is independent of the particular solution $u$. Now, if we choose $\varepsilon$ in equation (3.1) to be any value less than $\mu$, we see that $u-\rho>\varepsilon$ for the solutions constructed in this paper, and thus that we can remove the cut off function $h$ and replace the operator $\widetilde{Q}$ by $Q$.

Thus, there is a finite neighborhood of $\Xi_{a}$ in which the functions $u$ and $\rho$ we have found solve the original problem. A bound for the size of the neighborhood is given 
by $\left(u_{R}+1\right) / 2 \leq \rho \leq \xi_{a}$. When $a=\sqrt{2}$, then $\left(u_{R}+1\right) / 2=2$ and $\xi_{a}=5 / 2$, so the region in which we have solved the problem extends about one-third of the way down to the lower boundary, $\rho=1$.

This proves Theorem 3.1, and completes the proof of Theorem 1.1.

\section{REFERENCES}

[1] G. Ben-Dor, Shock Wave Reflection Phenomena, Springer-Verlag, New York, 1992.

[2] M. Brio and J. K. Hunter, Mach reflection for the two dimensional Burgers equation, Physica D, 60 (1992), pp. 194-207.

[3] M. BRio AND J. K. Hunter, Weak shock reflection, Journal of Fluid Mechanics, to appear.

[4] S. Čanić and B. L. KeYfitz, An elliptic problem arising from the unsteady transonic small disturbance equation, Journal of Differential Equations, 125 (1996), pp. 548-574.

[5] S. ČANIĆ AND B. L. KEYFITZ, A smooth solution for a Keldysh type equation, Communications in Partial Differential Equations, 21 (1996), pp. 319-340.

[6] S. Ćanić AND B. L. KeYFitz, Quasi-one-dimensional Riemann problems and their role in self-similar two-dimensional problems, Archive for Rational Mechanics and Analysis, 144 (1998), pp. 233-258.

[7] S. ČANIĆ AND B. L. KeYFITZ, Riemann problems for the two-dimensional unsteady transonic small disturbance equation, SIAM Journal on Applied Mathematics, 58 (1998), pp. 636665.

[8] S. Čanić, B. L. Keyfitz, and G. M. Lieberman, A proof of existence of perturbed steady transonic shocks via a free boundary problem, Communications on Pure and Applied Mathematics, LIII (2000), pp. 484-511.

[9] S. ČAnić, B. L. KeYfitz, AND D. H. WAGNER, A bifurcation diagram for oblique shock interactions in the unsteady transonic small disturbance equation, in Proceedings of the Fifth International Conference on Hyperbolic Problems: Theory, Numerics, and Applications, J. Glimm, et al., eds., World Scientific, Singapore, 1996, pp. 178-187.

[10] S. Ćanić AND D. Mirković, A numerical study of Riemann problems for the two-dimensional unsteady transonic small disturbance equation, SIAM Journal on Applied Mathematics, 58 (1998), pp. 1365-1393.

[11] L. GÅRDING, Some Points of Analysis and Their History, American Mathematical Society, Providence, 1997.

[12] D. Gilbarg AND L. Hörmander, Intermediate Schauder estimates, Archive for Rational Mechanics and Analysis, 74 (1980), pp. 297-318.

[13] D. Gilbarg and N. S. Trudinger, Elliptic Partial Differential Equations of Second Order, Springer-Verlag, New York, Second edition, 1983.

[14] G. M. Lieberman, The Perron process applied to oblique derivative problems, Advances in Mathematics, 55 (1985), pp. 161-172.

[15] G. M. Lieberman, Mixed boundary value problems for elliptic and parabolic differential equation of second order, Journal of Mathematical Analysis and Applications, 113 (1986), pp. $422-440$.

[16] G. M. Lieberman, Optimal Hölder regularity for mixed boundary value problems, Journal of Mathematical Analysis and Applications, 143 (1989), pp. 572-586.

[17] C. S. Morawetz, Potential theory for regular and Mach reflection of a shock at a wedge, Communications on Pure and Applied Mathematics, XLVII (1994), pp. 593-624.

[18] E. G. TABAK AND R. R. Rosales, Focusing of weak shock waves and the von Neumann paradox of oblique shock reflection, Physics of Fluids A, 6 (1994), pp. 1874-1892. 
